\title{
Intra-aortic Balloon Pump in Toxic Myocarditis Due to Aluminum Phosphide Poisoning
}

\author{
Latha M. Siddaiah, MBBS, Srilakshmi M. Adhyapak, DNB, Santosh M. Jaydev, DNB, Gurappa G. \\ Shetty, DM, Kiron Varghese, DM, Chandrakanth B. Patil, DM, Shamanna S. Iyengar, DM
}

Department of Cardiology, St. John's Medical College Hospital, Bangalore, India

\begin{abstract}
Introduction: Aluminum phosphide (ALP) is a pesticide that is highly toxic. It is a mitochondrial toxin that causes death by cardiac and metabolic toxicity. The most ominous effect is cardiac toxicity, which may range from minor electrocardiographic abnormalities to severe depression of cardiac contractility secondary to toxic myocarditis. There is no documented report of the use of Intra-aortic Balloon Pump (IABP) for toxic myocarditis from ALP poisoning, although it has been used effectively for toxic myocarditis due to other toxins.

Case Report: We are reporting a young female who presented with ALP poisoning, in cardiogenic shock due to myocarditis. We used an IABP for cardio-circulatory support until the effects of ALP resolved. She is the only reported survivor of ALP poisoning, presenting with cardiogenic shock.

Discussion: Several reports describe the use of IABP for cardiogenic shock due to toxic myocarditis. There is no report in the medical literature using IABP for cardiogenic shock caused by ALP toxin-induced myocarditis. Our patient presented with cardiocirculatory shock, necessitating the use of inotropes and mechanical ventilation. As she did not improve despite ventilation and maximal doses of inotropes, IABP was used for cardio-circulatory support until the effects of ALP resolved.
\end{abstract}

\section{INTRODUCTION}

Aluminum phosphide (ALP) is a highly toxic, solid fumigant pesticide used in the northern states of India $[1,2]$. It is the most commonly abused suicidal chemical agent in the state of Haryana, India, accounting for $75 \%$ to $80 \%$ of all poisoning deaths [1]. On contact with moisture, ALP releases phosphine, which is responsible for inhibition of mitochondrial cytochrome oxidase. Mortality is primarily due to cardiac and/or metabolic toxicity. As there is no specific antidote for ALP poisoning, only supportive care is available.

\section{CASE REPORT}

A 22-year-old woman presented to our emergency department (ED) 6 hours after ingestion of one 0.6-g tablet of quickphos (56\% aluminum phosphide). There was history of one episode of vomiting 2-3 hours after ingestion. She received gastric lavage at a local hospital and was referred to us for further management. She had no significant past medical history and was not on any medications. Her blood pressure was $80 / 60 \mathrm{mmHg}$, heart rate 128 beats/min, and respiratory rate was $18 / \mathrm{min}$.

Cardiovascular examination revealed S3 gallop, and lung

Keywords: aluminum phosphide, cardiogenic shock, intra-aortic balloon pump

Notes: There was no outside funding of any kind used for this study.

Corresponding Author: Dr. Latha M. Siddaiah, Department of Cardiology, St. John's Medical College Hospital, Sarjapura Road, Bangalore 560034, India.E-Mail: dr.latha_ms@yahoo.co.in or lathams_tulasi@rediffmail.com 
fields were clear. She brought a container of the toxin, thus a toxicological screen was not obtained. Her arterial blood gas (ABG) revealed the following: $\mathrm{pH} 7.321$; sodium bicarbonate (HCO3), 11.8 meq; base excess (BE), 14; and $\mathrm{SaO} 2,97 \%$. She was given 12 $\mathrm{mEq}$ IV sodium bicarbonate of $7.5 \%$ strength $(12 \mathrm{ml}, 1 \mathrm{mEq}=$ $1 \mathrm{ml}$ ) over 4 hours, started on IV fluids $0.9-\mathrm{N}$ normal saline at a rate of $100 \mathrm{ml} /$ hour $(2 \mathrm{~L} /$ day), inotrope infusion of dobutamine $2-4 \mu \mathrm{g} / \mathrm{kg}$ per minute, and noradrenaline $0.04 \mathrm{mg} / \mathrm{minute}$. Five hours after admission, oxygen desaturation occurred with worsening shock and the patient was intubated and received mechanical ventilation.

Admission electrocardiogram (ECG) showed sinus tachycardia with ST depression in II, III, AVF, V4-V6 (Figure 1). A chest radiograph showed a small right-sided pleural effusion. Echocardiography revealed normal valves, dilatation of all chambers, global hypokinesia, and left ventricular ejection fraction (LVEF) of 35\%. Her admission hemogram and biochemistry were normal. Her hemoglobin was $14.7 \mathrm{~g} / \mathrm{dl}$; total leukocyte count (TLC), 10,800 cells $/ \mathrm{mm}^{3}$; platelet count (PC), 2.2 cells $/ \mathrm{mm}^{3}$; blood urea, 26 $\mathrm{mg} / \mathrm{dl}$; creatinine, $0.7 \mathrm{mg} / \mathrm{dl}$; sodium, $141 \mathrm{mEq} / \mathrm{L}$; potassium, 4.1 $\mathrm{mEq} / \mathrm{L}$; chloride, $106 \mathrm{mEq} / \mathrm{L}$; International Normalized Ratio, 1.8; transaminases-ALT and AST-37 and 53 IU, respectively; troponin I, $0.05 \mathrm{IU}$ (normal 0.04). Serum calcium was $7.9 \mathrm{mg} / \mathrm{dl}$ and magnesium was $1.9 \mathrm{mg} / \mathrm{dl}$. These electrolyte abnormalities were corrected. Intravenous magnesium sulfate $2 \mathrm{~g}$ in 100-ml saline was administered over 1 hour and was repeated the next day. Intravenous unfractionated heparin was initiated at a dose of 1000
IU/hour as prophylaxis for deep vein thrombosis. Prophylactic broad spectrum antibiotics were administered: magnamycin 1-g IV every 6 hours and Amikacin 500-mg IV every 6 hours.

On day 5 of admission, the patient continued to be tachycardic [heart rate (HR),152/min] and hypotensive (systolic BP, $70 \mathrm{mmHg}$ ) with maximal doses of inotropes (dobutamine 15-20 $\mu \mathrm{g} / \mathrm{kg} / \mathrm{minute}$ and noradrenaline $0.8 \mathrm{mg}$ to $1 \mathrm{mg} / \mathrm{minute})$. Because of continued hypotension and signs of decreased perfusion (decreased urine output of $250 \mathrm{ml}$ in 24 hours), IABP was inserted after assessing its risks and benefits. On insertion of IABP, there was improvement in her BP to $110 / 70 \mathrm{mmHg}$, with a mean $\mathrm{BP}$ of $80 \mathrm{mmHg}$. Perfusion to vital organs improved, with an increase in urine output to $2.3 \mathrm{~L}$ /day. On day 8 , her $\mathrm{Hb}$, TLC, DC, PC, and serum potassium decreased ( $\mathrm{Hb} 7.8 \mathrm{~g} / \mathrm{dl}$, TC $8600 / \mathrm{mm}^{2}$, PC $41.000 / \mathrm{mm}^{2}$, and serum potassium was 3.9 $\mathrm{mEq} / \mathrm{L}$ ). Her liver function tests (LFT) were abnormal, with rises in AST, ALT, and alkaline phosphatase: $556 \mathrm{U} / \mathrm{L}, 356 \mathrm{U} / \mathrm{L}$, and $110 \mathrm{U} / \mathrm{L}$, respectively. Hypocalcemia and hypomagnesemia persisted. She was transfused with $2 \mathrm{U}$ of packed cells and platelet rich plasma and received IV potassium chloride $20 \mathrm{ml}$ (40 $\mathrm{mEq}$ ) of $15 \%$ strength in $500-\mathrm{ml}$ saline, magnesium sulphate 2 $\mathrm{g}$ in $100-\mathrm{ml}$ saline, and calcium gluconate $10 \mathrm{ml}$ (1.375 g calcium) in $10 \%$ strength.

On day 10 (i.e., day 5 of IABP), her BP stabilized at 120/60 $\mathrm{mmHg}$. Repeat echocardiogram revealed mild LV global hypokinesia with left ventricular ejection fraction (LVEF) of 35\% to $45 \%$. IABP was weaned and discontinued. On day 15 , she improved

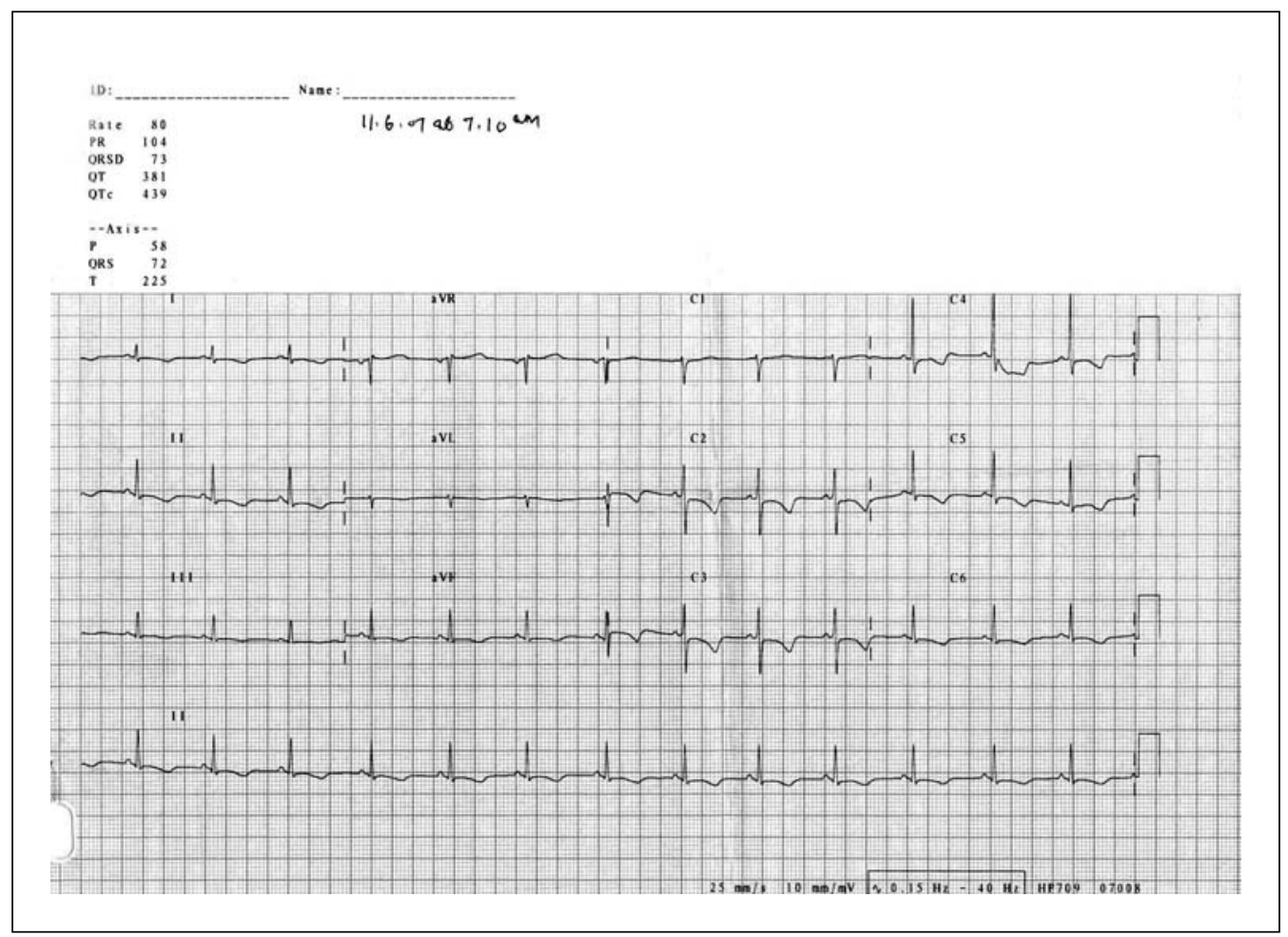

Figure 1: Admission electrocardiogram showing sinus tachycardia with ST segment depression in leads 2, 3, AVF, and V4-V6. 


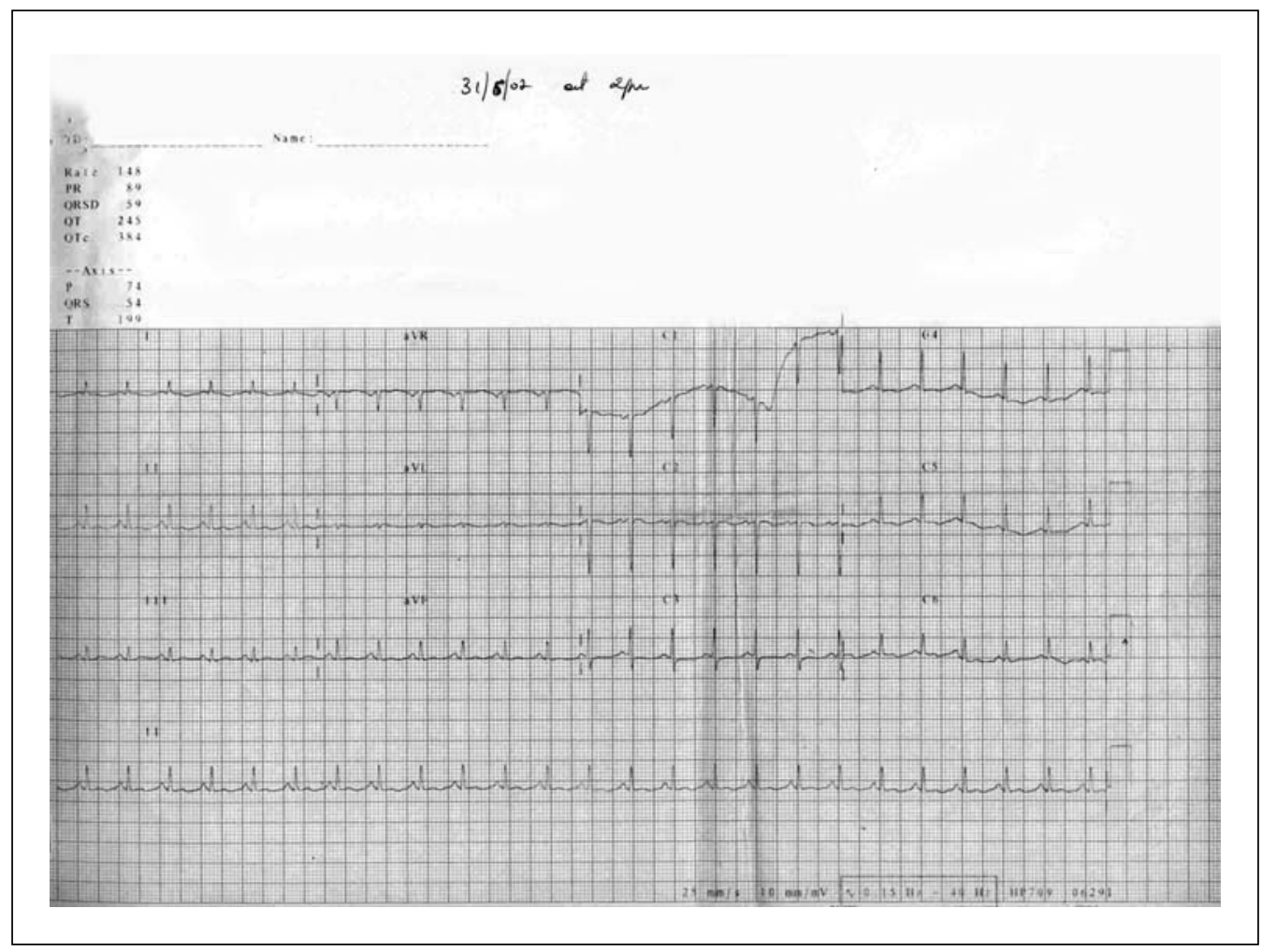

Figure 2: Electrocardiogram on day 16 showing sinus tachycardia with no significant ST segment or $T$ wave changes.

clinically with heart rate of $92 / \mathrm{min}$, BP $128 / 68 \mathrm{mmHg}$, and good urine output. She was extubated on the same day, and inotropes were tapered and stopped. Her hematological and biochemical parameters normalized. Echocardiogram on day 16 of admission showed normal chambers and LVEF of $65 \%$. The ECG on day 16 of admission showed sinus tachycardia with no significant ST segment or T wave abnormalities (Figure 2). She subsequently recovered and was discharged on day 22 of hospitalization.

\section{DISCUSSION}

ALP is a synthetic substance with a garlic odor, available as tablets (3 g) and pellets $(0.6 \mathrm{~g})[1,2]$, with trade names such as Quickphos, Celphos, and Alphos. The fatal dose for an adult is $0.5 \mathrm{~g}[2,3]$. On contact with moisture, ALP releases phosphine, which inhibits cytochrome oxidase of mitochondria, blocking the electron transfer chain and oxidative phosphorylation. It also inhibits catalase and induces superoxide dismutase in humans, leading to free radical stress, causing hypoxic cell injury [4].

Clinical features include gastrointestinal disturbances, shock, acute respiratory distress syndrome, acute renal failure, hemorrhage, disseminated intravascular coagulation, acidosis, and cerebral anoxia [2].
The most ominous clinical manifestation is cardiovascular toxicity in the form of toxic chemical myocarditis, leading to severe left ventricular dysfunction $[5,6]$. Minor ECG changes such as ST segment elevation or depression, PR prolongation, broad QRS complexes, right bundle branch block (RBBB), left bundle branch block (LBBB), complete heart block (CHB), supraventricular ectopic beats, or atrial fibrillation may occur [5].

Biochemical abnormalities including elevated AST, creatine phosphokinase (CPK MB), and lactate dehydrogenase (LDH) can also occur. Diagnosis is by definitive history or silver nitrate test [1].

To date there is no antidote available for ALP poisoning [2)], the mainstay of treatment being supportive care. Gastric lavage using saline, sodium bicarbonate, potassium permanganate, and coconut oil have been reported to be effective in anecdotal reports (7). Shock has been managed in various centers with IV saline, dopamine, and parenteral hydrocortisone [2]. Conventional drugs such as digoxin, lidocaine, and atropine are not useful to treat ALP-induced arrhythmias [4]. Instead, magnesium sulfate is effective in both tachyarrhythmias and bradyarrhythmias, due to its membrane-stabilizing properties. Magnesium sulfate may be given in all cases of ALP poisoning irrespective of serum magnesium levels [4].

The use of IABP has been documented in severe LV dysfunction due to toxic myocarditis of other causes $[8,9]$. IABP decreases 
the afterload and improves perfusion to the vital organs and coronary arteries. It provides cardio-circulatory support and is useful as a bridge to recovery. The contraindications to its use are aortic dissection, moderate to severe aortic regurgitation, aortic aneurysms, peripheral vascular disease, neurological deficits, and sepsis, none of which were present in our patient. ALP is a lethal toxin and when myocarditis occurs, death rapidly ensues. Acute cardiovascular collapse is the most common mode of presentation seen in $60 \%$ to $100 \%$ of cases [3]. There is no report to date of patients surviving toxic myocarditis due to ALP poisoning. The use of IABP in these cases has not been documented, as these cases deteriorate to cardiac standstill almost immediately. Our patient presented with cardiogenic shock, where IABP was used as a bridge to recovery. Her recovery could be due to absorption of sublethal amounts of ALP, as she had vomited 2-3 hours after ingestion of the toxin.

One other interesting finding in our case was pancytopenia. According to current literature, ALP causes hemorrhage, disseminated intravascular coagulation (DIC), and hemolysis (hemolysis is more common with associated G-6-P deficiency) [1]. Our patient did not have any clinical features of hemorrhage, DIC, or hemolysis. We did not document elevated D-dimer or fibrinogen levels. Only the prothrombin time was prolonged. ALP being a mitochondrial toxin might cause cell injury directly (all cell lines including erythrocytes, leukocytes and thrombocytes) or it might act as a bone marrow depressant. Pancytopenia in our patient could be the result of either cell injury or bone marrow suppression.

\section{CONCLUSION}

IABP has been useful as a cardiocirculatory assist device in a patient with circulatory collapse secondary to severe toxic myocarditis caused by ALP poisoning. This intervention resulted in improvement of cardio-circulatory shock, accompanied by im- provement in cardiac function as the toxic effects of ALP resolved.

The authors have no potential financial conflicts of interest to report.

\section{REFERENCES}

1. Gupta S, Ahlawat SK. Aluminum phosphide poisoning. A Review. J Toxicol/Clin Toxicol 1995;33:19-24.

2. Hajouji Idrissi M, Oualili L, Abidi K, et al. Severity factors of aluminum phosphide poisoning (Phostoxin). Ann Fr Anesth Reanim 2006;25:382-385.

3. Tripathi SK, Gautam CS, Sharma PL. Clinical pharmacology of aluminium phosphide poisoning. Indian J Pharmacol 1992;24:134-137.

4. Chugh N. Aluminium phosphide. In: Lall SB, ed. Essentials of clinical toxicology. New Delhi: Narosa Publishing House, 1998:41-46.

5. Singh RB, Rastogi SS, Singh DS. Cardiovascular manifestations of aluminum phosphide intoxication. J Assoc Physicians India 1989;37:590-592.

6. Akkouui M, Achur S, Abidi K, et al. Reversible myocardial injury associated with aluminum phosphide poisoning. Clin Toxicol 2007; 45:728-731.

7. Shadnia S, Rahimi M, Pajoumand A, et al. Successful treatment of acute aluminium phosphide poisoning: possible benefit of coconut oil. Hum Exp Toxicol 2005;24(4):215-218.

8. Uriel N, Kaluski E, Hendler A, et al. Cardiogenic shock in a young female with multiple sclerosis. Resuscitation 2006;70(1):153-157.

9. David JS, Gueugniaud PY, Hepp A, et al. Severe heart failure secondary to 5-fluorouracil and low doses of folinic acid: usefulness of an intra-aortic balloon pump. Crit Care Med 2000;28(10): 3558-3560. 